\title{
Expression of the melittin gene of Apis cerana cerana (Hymenoptera: Apidae) in insect cells
}

\author{
WAN-JUN SHI ${ }^{1,2}, \mathrm{JIA}_{\mathrm{IAN}} \mathrm{CHENG}^{1 *}$ and CHUAN-XI ZHANG ${ }^{1 *}$ \\ ${ }^{1}$ Institute of Insect Sciences, Zhejiang University, 310029 Hangzhou, China \\ ${ }^{2}$ Department of Resources and Environmental Science, Shanghai Jiao Tong University, 201101 Shanghai, China; \\ e-mails: wjshi@sjtu.edu.cn; chxzhang@zju.edu.cn; jacheng@sun.zju.edu.cn
}

Key words. Hymenoptera, Apidae, Apis cerana cerana, Chinese honeybee, melittin, insect cells, protein expression

\begin{abstract}
A fragment encoding melittin cDNA from Apis cerana cerana fused with glutathione S-transferase gene was inserted into the multiple cloning site of the pBacFastHTb to construct a recombinant donor plasmid, pBacHT-GSTAccM, which was transposed to the target bacmid in E. coli (DH10) by Tn7 transposition function. Then the recombinant baculovirus Bacmid-GSTAccM was transfected into Tn-5B1-4 cells of the cabbage looper, Trichoplusia ni, mediated by lipofectin. The expressed protein of about 34 $\mathrm{kDa}$ was detected by Western blotting and triple antibody sandwich ELISA, indicating that the recombinant protein is the fusion protein of GSTAccM. Thin layer scanning showed that the expression level of GSTAccM was about 7\% of the total cell protein. Purified and recovered recombinant melittin of A. c. cerana showed bioactivity in activating rabbit platelets to aggregate.
\end{abstract}

\section{INTRODUCTION}

The honeybee, Apis mellifera L., venom gland produces an aqueous secretion, which contains a significant quantity of two enzymes and four peptides. The enzymes are phospholipase $\mathrm{A}_{2}$ and hyaluronidase, and the peptides are melittin, apamin, MCD, and secapin (Zhang et al., 2003). Melittin, comprising 50\% of its dry weight, is a basic, amphipathic polypeptide (Pott et al., 1998). It is characterized by its powerful hemolytic activity and a popular model peptide for probing lipid-peptide interactions (Subbalakshmi et al., 1999; Benachir et al., 1997). In nanomolar amounts, melittin can form ion channels, whereas at higher concentrations it causes membrane disruption that leads to cell lysis (Lam et al., 2001). Purification from the bee venom and chemical synthesis of melittin are the only current approaches to obtaining melittin. The purification approach is often complicated by the presence of phospholipase $A_{2}$ in the samples, whereas chemical synthesis of melittin is very expensive, which limits any extensive application and scientific research on melittin. In our previous report, the melittin gene of the Chinese honeybee, Apis cerana cerana Fabricius (Hymenoptera: Apidae), was shown to be highly expressed in E. coli using the pGEX expression system (Shi et al., 2004), however, about $60 \%$ of the expressed products were inclusion bodies and it was very difficult to change these inclusion bodies into the active form. Therefore, we explore eukaryotic expression systems based on insect cells for the production of melittin of the Chinese honeybee as these systems have the potential for producing large amounts of correctly processed and secreted proteins. Here we report the successful expression of the melittin gene from the Chinese honeybee as a glutathione S-transferase (GST) fusion protein in baculovirus-infected insect cells.

\section{MATERIAL AND METHODS}

Honeybee (A. c. cerana) workers were obtained from the beekeeping farm of Zhejiang University. The bacterial strain, DH10Bac, Bac-to-Bac ${ }^{\circledR}$ Baculovirus expression vector, Tn5B1-4 cells of the cabbage looper, Trichoplusia ni (Hübner), were products of Invitrogen (California, USA). The recombinant plasmid pGEX-AccM was kept in the Molecular Laboratory of our Institute.

Restriction endonucleases, X-gal, IPTG, DL-2000, DL-15000 markers came from Takara (Dalian, China). Taq polymerase, NBT/BCIP, nitrocellulose, TEMED, kanamycin, tetracyclin and genlamycin were purchased from Sangon (Shanghai, China). Goat anti rabbit IgG-HRP were purchased from Sino-American Biotechnology Co. (Shanghai, China). Standard natural melittin of $A$. mellifera, ovalbumin (OVA), TNM-FH insect medium and thrombin protease came from Sigma (Steinheim, Germany). pGEM®-T easy vector was manufactured by Promega (Madison, USA). The PCR purification kit was from Qiagen (Shanghai, China), $\mathrm{TXB}_{2}$ RIA kit was from Furui Co. (Beijing, China), goat anti GST antibody and GST 96-well detection module and MicroSpin ${ }^{\mathrm{TM}}$ GST purification module were from Amersham Biosciences UK Limited (Buckinghamshire, England).

Cloning of the melittin cDNA and glutathione S-transferase (GST) fusion gene

A fragment encoding melittin cDNA and glutathione S-transferse was amplified by PCR from the recombinant plasmid pGEX-AccM using the forward primer FP1-GST (5'GAATCTAGAATGTCCCCTATACTAGGTT) and the reverse primer RP2-AccM (5'-GAAGCTTCTAACCCTGTTGCCTC TT). FP1-GST was designed from the GST gene sequence of the plasmid pGEX-AccM, whereas the reverse primer RP2-AccM was designed from A. c. cerana melittin cDNA (Shi et al., 2003, 2004). Amplification was performed in a ThermoHybaid thermocycler (Hybaid Limited, UK) with one cycle of $94^{\circ} \mathrm{C}$ for 3 min followed by 30 cycles of denaturation $\left(60 \mathrm{~s}\right.$ at $\left.94^{\circ} \mathrm{C}\right)$, annealing $\left(60 \mathrm{~s}\right.$ at $\left.54^{\circ} \mathrm{C}\right)$ and extension $\left(90 \mathrm{~s}\right.$ at $\left.72^{\circ} \mathrm{C}\right)$ and a final step of $72^{\circ} \mathrm{C}$ for $10 \mathrm{~min}$. PCR products were visualized on a $1.0 \%$ ethidium bromide stained gel. After purification with the QIAquick purification kit these PCR products were subcloned into the pGEM ${ }^{\circledR}-\mathrm{T}$ easy vector, and plasmids pGEM $\mathbb{R}-G S T A c c M$ were obtained.

\footnotetext{
* Corresponding authors.
} 


\section{Generation of recombinant baculovirus}

The recombinant plasmid pGEM $®$-GSTAccM was digested with XbaI/HindIII endonucleases and ligated into a pBacFastHTb vector cut with $X b a \mathrm{I} / H$ indIII. This final recombinant baculovirus expression vector pBacHT-GSTAccM was identified by PCR with primers FP1-GST and RP2-AccM. After being transformed into $E$. coli $\mathrm{DH} 10 \mathrm{Bac}$ cells, the recombinant baculovirus Bacmid-GSTAccM was checked by PCR with PUC/M13 primers (Bacmid-FP1 primer: 5'-GTAAAACGACG GCCAGT; Bacmid-RP2 primer: 5'-AACAGCTATGACCATG) designed from the left and right arms sequences of the bacterial transposon Tn7 (mini-att Tn7) and GST-AccM primers (FP1-GST and RP2-AccM), respectively.

\section{Transfection of Tn-5B1-4 cells with recombinant bacmid DNA}

Tn-5B1-4 cells were seeded at $1 \times 10^{5}$ per $35-\mathrm{mm}$ well in $2 \mathrm{ml}$ of TNM-FH insect medium containing $10 \%$ fetal bovine serum $10 \mathrm{~h}$ before transfection. For each transfection, $1 \mu \mathrm{g}$ bacmid DNA and $20 \mu 1$ lipofectin reagent were diluted with $50 \mu 1$ and $30 \mu \mathrm{lddH_{2 }} \mathrm{O}$, respectively, and these two solutions were subsequently combined and incubated at room temperature for $45 \mathrm{~min}$ to allow lipid-DNA complexes to form. After the Tn-5B1-4 cells were washed with $2 \mathrm{ml}$ of TNM-FH insect medium without fetal bovine serum thrice, $0.9 \mathrm{ml}$ of TNM-FH insect medium was added into the tube containing the lipid-DNA complex, and the mixtures were overlaid onto the cells. After the cells were incubated at $27^{\circ} \mathrm{C}$ for $5 \mathrm{~h}$, the medium was removed and replaced with $2 \mathrm{ml}$ of TNM-FH insect medium containing $10 \%$ fetal bovine serum. The cells were incubated at $27^{\circ} \mathrm{C}$ for $96 \mathrm{~h}$. The viruses were harvested from the cell medium by centrifugation for $5 \mathrm{~min}$ at $5000 \mathrm{~g}$. The supernatant was then transferred to a fresh tube and ready for future infection. The recombinant baculovirus Bacmid-GSTAccM DNA extracted from the cells was checked by PCR using GST-AccM primers and PUC/M13 primers, respectively.

\section{Expression of GSTAccM fusion protein in insect cells}

Tn-5B1-4 cells, infected with Bacmid-GSTAccM or Bacmid at multiplicity of infection of 10 , were prepared. The cultures were maintained in TNM-FH insect medium at $27^{\circ} \mathrm{C}$ for 4 days; at the same time the cytopathic effects (CPE) were observed under the microscope. After cultivation, the transfected Tn5B1-4 cells were harvested and centrifuged at $1000 \mathrm{~g}$ for 10 min. The cell pellet was washed with $1 \times$ PBS thrice and then boiled for $5 \mathrm{~min}$ after addition of $2 \times$ SDS-PAGE loading buffer. Expression level of recombinant protein GSTAccM was monitored by $12 \%$ SDS-PAGE stained with Coomassie brilliant blue $\mathrm{R}-250$.

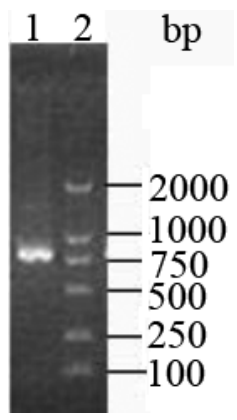

Fig. 1. PCR product of GSTAccM. Lane 1, Apis cerana cerana fragment of the melittin cDNA and GST fusion gene; lane 2, DNA size marker.

\section{Immunoblot analysis by Western blotting}

After electrophoresis on a $12 \%$ SDS-PAGE, the proteins were transferred onto two nitrocellulose membranes using a Bio-Rad transblotter. One membrane was incubated with rabbit antimelittin (A. mellifera) antibodies and goat anti-rabbit IgG-HRP, whereas the other membrane was probed with goat anti-GST antibodies and rabbit anti-goat IgG-HRP, according to manufacturer's protocol.

\section{Triple antibody sandwich ELISA}

After sonication, the expressed protein was pipetted into each well of a GST 96-well detection plate, which had been coated with goat anti-GST antibody to capture GST fusion proteins by the supplier (Pharmacia) in advance. The captured fusion protein was then incubated with the secondary antibody, the rabbit anti-melittin antiserum. Finally, the antibody-antigen-antibody complex was detected with a third antibody, the goat anti-rabbit IgG-HRP, using the method based on manufacturer's instructions.

\section{Assay of the melittin activity}

The bioactivity of the heterologously expressed melittin was evaluated based on the method of activation of melittin from $A$. mellifera venom to rabbit platelets as reported by Yu \& Xiong (1996). The experiment was carried out in three groups: (1) control, $0.9 \mathrm{ml}$ rabbit platelets $+0.1 \mathrm{ml} 0.9 \% \mathrm{NaCl}$; (2) melittin, 0.9 $\mathrm{ml}$ rabbit platelets + purified and recovered recombinant melittin $+0.1 \mathrm{ml} 0.9 \% \mathrm{NaCl}$; and (3) standard, $0.9 \mathrm{ml}$ rabbit platelets + melittin of $A$. mellifera $+0.1 \mathrm{ml} 0.9 \% \mathrm{NaCl}$. The amount of thromboxane $\mathrm{B}_{2}$, which was produced when rabbit platelets aggregated under activation of melittin, was detected.

\section{RESULTS}

\section{Expression of GSTAccM in insect cells}

A 769-bp fragment of the melittin cDNA and GST fusion gene was amplified from a previously sequenced melittin recombinant plasmid pGEX-AccM DNA of A. c. cerana using PCR (Fig. 1) and cloned into pBacFastHTb donor vector. The fusion gene was further inserted into viral DNA by transposition, generating the recombinant baculovirus BacmidGSTAccM. Tn-5B1-4 cells were collected at 4 days postinfection of Bacmid-GSTAccM. The expressed protein band of about $34 \mathrm{kDa}$, which was consistent with the expected molecular weight of the fusion protein, GSTAccM, appeared on the SDS-

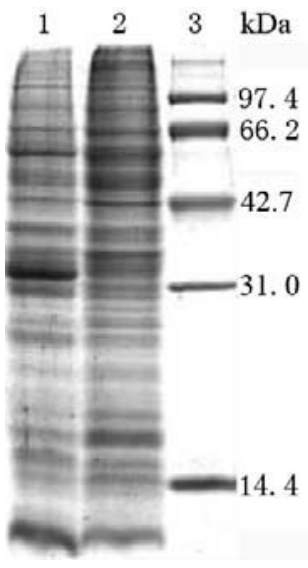

Fig. 2. SDS-PAGE analysis of GSTAccM expression products in Tn cells. Lane 1, sample from Tn cells infected with Bacmid-GSTAccM; lane 2, sample from Tn cells infected with Bacmid; lane 3, protein size marker. 


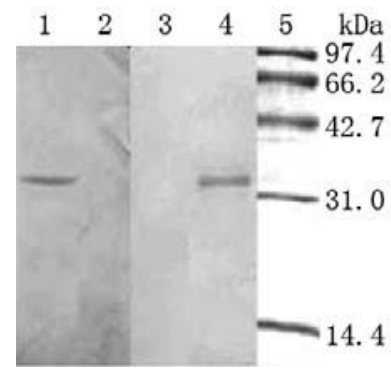

Fig. 3. Western blot analysis for GSTAccM expression products in Tn cells. Lane 1, expressed product probed with the serum immunized with melittin binding OVA antigen; lanes 2 and 3, samples from Tn cells infected with Bacmid; lane 4, expressed product probed with anti-GST antibody; lane 5, protein size marker.

PAGE profile (Fig. 2). Thin layer scanning showed that the expressed protein accumulated up to about $7 \%$ of total protein of insect cells.

\section{Western blotting and triple antibody sandwich ELISA}

Supernatant and cells of infected Tn-5B1-4 cells were collected and analyzed by Western blotting using a rabbit antimelittin antibody and goat anti-GST antibody, respectively. The proteins were both recognized by these two antibodies (Fig. 3), whereas the control Tn-5B1-4 cells infected with Bacmid did not produce a signal on immunoblot. It was proved that this product of expression was the expected fusion protein GSTAccM. After sonication each sample was also identified by triple antibody sandwich ELISA (Fig. 4). The absorbance at 490 nm of Tn-5B1-4 infected with recombinant baculovirus BacmidGSTAccM is two times higher than that of the control Tn cells infected Bacmid using the anti-melittin binding OVA antibody and anti-GST antibody at $1: 32000$ dilution. The result further confirmed that the expressed product was the GSTAccM fusion protein.

\section{Assay of bioactivity of the recovered recombinant melittin}

Recombinant AccM was expressed in insect cells as a fusion protein containing GST for affinity purification. The purified GSTAccM fusion protein up to $>90 \%$ of purity was obtained in a single step from the total proteins using affinity chromatog-

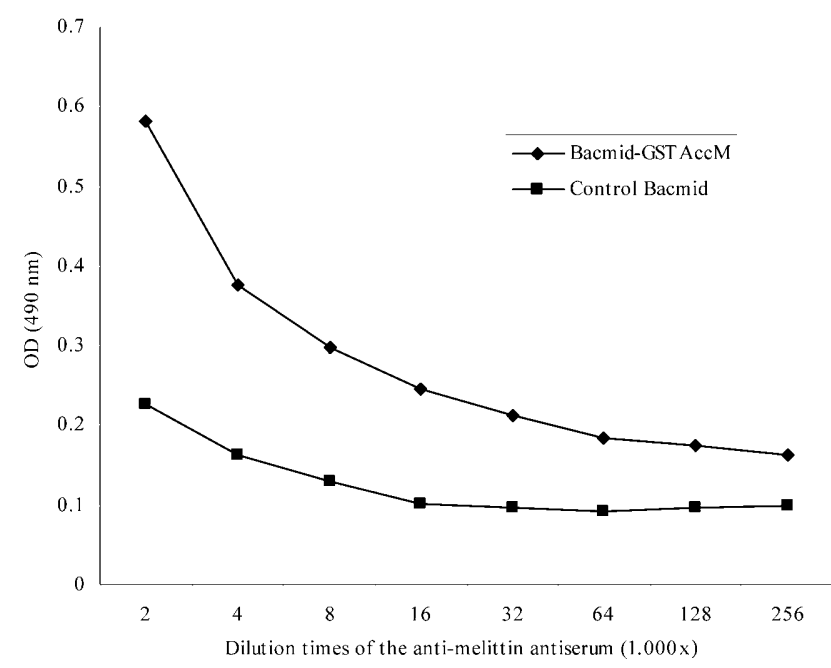

Fig. 4. Data of expression products in insect cells by triple antibody sandwich ELISA.

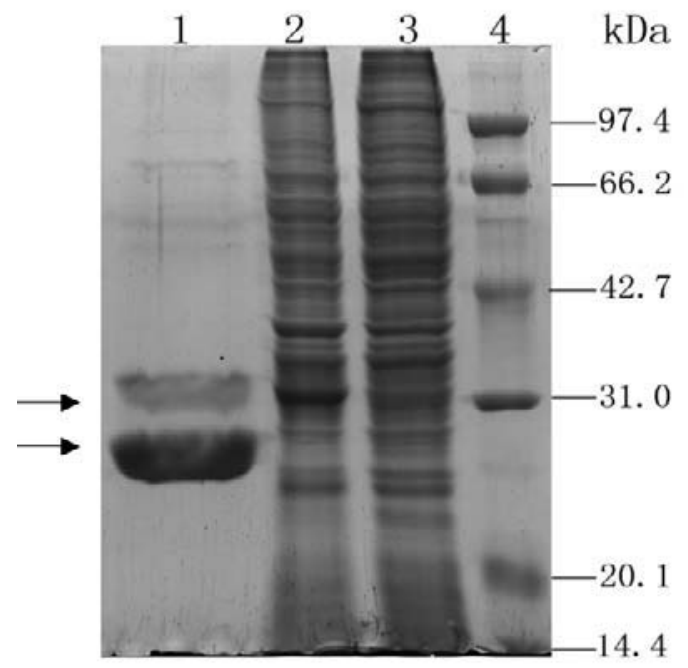

Fig. 5. SDS-PAGE analysis for proteolysis of purified GSTAccM protein. Lane 1, partial proteolysis of the purified GSTAccM protein (the upper arrow indicates the purified GSTAccM fusion protein; the nether arrow indicates the GST-tag after the cleavage of the fusion protein); lane 2, sample from Tn cells infected with Bacmid-GSTAccM; lane 3, sample from Tn cells infected with Bacmid; lane 4, protein size marker.

raphy of glutathione sepharose 4B. After the cleavage of the recombinant fusion protein with thrombin protease at $22^{\circ} \mathrm{C}$ for more than $16 \mathrm{~h}$, the GST was removed by affinity chromatography of glutathione sepharose $4 \mathrm{~B}$ and the recombinant melittin was recovered. The results are shown in Fig. 5. The recovered melittin was then used for the assay of bioactivity. Melittin bioactivity in activating aggregation of rabbit platelets was accompanied by the formation of thromboxane $B_{2}$. The results of formation of thromboxane $\mathrm{B}_{2}$ of melittin activating rabbit platelets are shown in Table 1. The results demonstrated that the recombinant melittin exhibited similar bioactivity as the natural melittin of $A$. mellifera.

TABLE 1. Formation of thromboxane $\mathrm{B}_{2}$ of melittin activating rabbit platelets.

\begin{tabular}{lccc}
\hline & $\begin{array}{c}\text { A.c. cerana } \\
\text { melittin }(51 \mathrm{mg} / \mathrm{L})\end{array}$ & $\begin{array}{c}\text { Standard melittin } \\
(51 \mathrm{mg} / \mathrm{L})\end{array}$ & Control \\
\hline $\mathrm{TXB}_{2}(\mathrm{ng} / \mathrm{L})$ & $458.99 \pm 1.8$ & $431.24 \pm 1.8$ & $378.23 \pm 1.8$ \\
\hline
\end{tabular}

\section{DISCUSSION}

The melittin gene of the Chinese honeybee was successfully expressed in insect cells using the Bac-to-Bac ${ }^{\circledR}$ baculovirus expression system. Compared with that of the melittin gene of the Chinese honeybee expressed in E. coli using the pGEX expression system (Shi et al., 2004), the expression product in this study is almost soluble. Here recombinant AccM was expressed in insect cells as a fusion protein containing GST because melittin has powerful hemolytic activity. AccM is easy to retrieve from the fusion protein. For example, Amersham Biosiences Company provides an integrated system for the purification, detection of fusion proteins containing GST and cleavage of the desired protein from GST. Our results showed that the use of Bac-to-Bac ${ }^{\circledR}$ baculovirus expression system and GST-tag are a good method for expression and purification of the melittin of $A$. cerana cerana. It is very simple to purify the expression product and recover the recombinant melittin. 
Moreover, the proteins can be purified to $>90 \%$ in a single step, which preserve antigenicity and functionality of the proteins.

As reported in the previous study, the prepromelittins from two honeybee species, $A$. mellifera and A. c. cerana, share more than $92 \%$ identities in amino acid sequences, whereas their melittins, located in the $\mathrm{COOH}$-terminal of the polypeptide chain (residues 44 70), share 100\% identities in amino acid sequences (Shi et al., 2003). Thus, the venom melittin protein of A. mellifera purchased from Sigma Co. was used as antigen to produce antibody for this experiment. The expression product of melittin gene from A. c. cerana was identified by its antiserum. The expression product was confirmed by Western blotting and triple antibody sandwich ELISA.

Purified and recovered recombinant melittin of A. c. cerana showed bioactivity in activating rabbit platelets to aggregate, accompanying the formation of thromboxane $\mathrm{B}_{2} .5-\mathrm{HT}$ released during the aggregation could aid the aggregation of the rabbit platelets, but the detailed mechanism of melittin activating rabbit platelets to aggregate is not clear yet.

Melittin is a cytolytic peptide extracted from bee venom (see Habermann, 1972, for a review). Bees have highly specialized cells to avoid melittin toxicity by melittin liberated from prepromelittin through several processing steps (Vlasak et al., 1983). In our study, the melittin from A. c. cerana was expressed successfully in the Baculovirus expression system. This approach may provide a valuable method for the production of the honeybee venom melittin through a eukaryotic gene expression system and thus makes recombinant melittin available for further study of its activity and applications.

ACKNOWLEDGMENTS. This study was supported by the National Natural Science Foundation of China (No. 30271008) and a foundation from the School of Agriculture and Biology, Shanghai Jiao Tong University (No. AE150054). We are grateful to Shu S.-K. and Cheng S.-L. for the supply of $A$. $c$. cerana.

\section{REFERENCES}

Benachir T., Monette M. \& Grenier J. 1997: Melittin-induced leakage from phosphatidylcholine vesicles is modulated by cholesterol: a property used for membrane targeting. Eur. Biophys. J. 25: 201-210.

Habermann E. 1972: Bee and wasp venoms. Science 177: 314-322.

Lam Y.H., Wassall S.R., Morton C.J., Smith R. \& Separovic F. 2001: Solid-state NMR structure determination of melittin in a lipid environment. Biophys. J. 81: 2752-2761.

Pott T., Paternostre M. \& Dufourc E.J. 1998: A comparative study of the action of melittin on sphingomyelin and phosphatidylcholine bilayers. Eur. Biophys. J. 27: 237-245.

Shi W.-J., Zhang S.-F., Zhang C.-X. \& Cheng J.-A. 2003: Cloning and sequencing of cDNA encoding prepromelittin in Apis cerana cerana and Apis mellifera. Acta Entomol. Sin. 46: 254-258.

ShI W.-J., Xu H.-J., Cheng J.-A. \& Zhang C.-X. 2004: Expression of the melittin gene of Apis cerana cerana in Escherichia coli. Protein Expres. Purif. 37: 213-219.

Subbalakshmi C., Nagaraj R. \& Sitaram N. 1999: Biological activities of C-terminal 15-residue synthetic fragment of melittin: design of an analog with improved antibacterial activity. FEBS Lett. 448: 62-66.

Vlasak R., Unger-ullmann C., Kreil G. \& Frischauf A.-M. 1983: Nucleotide sequence of cloned cDNA coding for honeybee prepromelittin. Eur. J. Biochem. 135: 123-126.

Yu X.D. \& Xiong Y.L. 1996: The activation of Melittin from Apis mellifera venom to rabbit platelets. J. Chongqing Teachers College (Nat. Sci.) 13(1): 15-20.

Zhang S.-F., Shi W.-J., Cheng J.-A. \& Zhang C.-X. 2003: Cloning and comparison of the genes encoding preproapamin from the venom of 2 honeybee and 4 wasp species. Entomol. Sin. 10: $21-26$.

Received August 23, 2005; revised and accepted March 3, 2006 